\title{
Is scheduled second chemoembolization necessary for early stage hepatocellular carcinoma showing complete response after first chemoembolization?
}

\author{
Jin Wook Chung \\ Department of Radiology, Seoul National University Hospital, Seoul National University College of Medicine, Seoul, Korea
}

Keywords: Hepatocellular carcinoma; Chemoembolization

\section{See Article on Page 42}

The reported complete response rate after single session of chemoembolization for small hepatocellular carcinomas ranged from $75 \%$ to $80 \%$ on mRECIST evaluation of imaging studies. ${ }^{1,2}$ However, considerable proportion of tumor nodules with complete initial response after chemoembolization show local tumor progression during long-term follow-up. The cumulative 5-year local tumor progression rate of chemoembolization for early stage hepatocellular carcinomas was reported to be as high as $73 \%{ }^{2}$

There is a general belief that better local tumor control can lead to longer survival. Therefore, many physicians are enthusiastic to achieve complete local tumor control for early stage hepatocellular carcinomas by applying combination strategy or potent regimen. However, we have to remember that most of patients who receive chemoembolization have underlying liver cirrhosis and portal hypertension. In patients with portal hypertension, local tumor control and preservation of normal liver parenchyma is equally important (should be well balanced) because remote site recurrence is very common and multiple treatments are usually required during the course of the disease. Unfortunately, better local tumor control means greater hepatic parenchymal loss in general. Local tumor control rate is highest in hepatic resection followed by thermal ablation and chemoembolization. On the contrary, normal liver parenchymal damage or major adverse events are smallest in chemoembolization followed by thermal ablation and resection.' As a result, retrospective comparison with propensity score matching revealed that there was no significant difference in long-term survival between hepatic resection and thermal ablation and chemoembolization in small hepatocellular carcinomas. In addition, recent large retrospective series for local thermal ablation also revealed that local tumor progression was not a significant prognostic factor for survival. ${ }^{3,4}$

Because of common local tumor progression after chemoembolization, scheduled $2^{\text {nd }}$ chemoembolization would be a reasonable option. Recently, the results of one randomized trial evaluating the role of additional angiography and chemoembolization in

\section{Abbreviations:}

\section{Corresponding author : Jin Wook Chung}

Department of Radiology, Seoul National University Hospital, Seoul National University College of Medicine, 101 Daehak-ro, Jongno-gu, Seoul 03080, Korea

Tel: +82-2-2072-2584, Fax: +82-2-743-6385

E-mail: chungjw@snu.ac.kr 
patients with hepatocellular carcinomas who achieved complete necrosis following chemoembolization were published. ${ }^{5}$ In that trial, $15 \%$ of patients who underwent scheduled $2^{\text {nd }}$ chemoembolization had angiographic evidence of residual viable tumor and treated early. However, the cumulative recurrence rate and overall survival rates at 1- and 2-year was not significantly different between the two groups. In addition, early randomized trials comparing chemoembolization with best supportive care in advanced stage hepatocellular carcinomas failed to demonstrate survival advantage of chemoembolization and were criticized for adopting scheduled chemoembolization regardless of tumor response which might have negative impact on survival by accelerating liver failure. Therefore, consensus opinion for repeating chemoembolization is on demand rather than scheduled.

In the current issue, Kim et al. conducted a retrospective study comparing scheduled repetition of chemoembolization with on demand approach. ${ }^{6}$ In multivariate analysis, scheduled repetition of chemoembolization was an independent favorable prognostic factor for survival. However, in the manuscript, there were no supporting data how scheduled repetition of chemoembolization led to survival improvement. How many patients had residual viable tumor on scheduled $2^{\text {nd }}$ chemoembolization? Was scheduled $2^{\text {nd }}$ chemoembolization significantly decreased the rate of locally invasive recurrence or chemoembolization failure? Was scheduled $2^{\text {nd }}$ chemoembolization decreased remote intrahepatic recurrence or distant metastasis rate? They reported that treatment strategy (scheduled vs. on-demand) was not associated with recurrencefree survival (39.7\% vs. $49.2 \%$ at 1 year, $P=0.26)$ and local recurrence-free survival $(68.3 \%$ vs. $66.8 \%$ at 1 year, $P=0.38)$. Retrospective series are prone to selection bias. I believe there are multiple hidden factors which may affect $2^{\text {nd }}$ treatment strategy: tumor morphology, lipiodol uptake pattern, changes in tumor markers, age, performance, compliance, socioeconomic factors, etc. If there were physicians who preferred and stuck to scheduled chemoembolization, it would be better to see the survival difference between physician groups. The treatment strategies for recurrent tumor also may affect long-term survival. Therefore, it should be reported how the recurrent tumors were treated. If chemoembolization was selected for recurrent tumors, it should be mentioned whether scheduled chemoembolization principle was applied to the recurrent tumors. Finally, the influence of scheduled chemoembolization on hepatic function should be analyzed and reported.

Because routine scheduled chemoembolization for complete radiological response may increase the number of procedure and procedural costs and have negative impact on hepatic functional reserve, it should be critically evaluated with further randomized trial.

Finally, local tumor progression rates after chemoembolization for small hepatocellular carcinomas largely depended on the ultraselective catheterization of all tumor feeders and adequate endpoint of the procedure. ${ }^{7,8}$ To reduce local tumor recurrence, it is important to utilize recent technological advances of $\mathrm{C}$-arm cone-beam CT and small-bore microcatheters and keep the principle of "as selective as possible" and "oily portogram" as the endpoint of the procedure in daily practice.

\section{Conflicts of Interest}

The author has no conflicts to disclose.

\section{REFERENCES}

1. Yang HJ, Lee JH, Lee DH, Yu SJ, Kim YJ, Yoon JH, et al. Small singlenodule hepatocellular carcinoma: comparison of transarterial chemoembolization, radiofrequency ablation, and hepatic resection by using inverse probability weighting. Radiology 2014;271:909-918.

2. Kim JW, Kim JH, Sung KB, Ko HK, Shin JH, Kim PN, et al. Transarterial chemoembolization vs. radiofrequency ablation for the treatment of single hepatocellular carcinoma $2 \mathrm{~cm}$ or smaller. Am J Gastroenterol 2014;109:1234-1240.

3. Kim YS, Lim HK, Rhim H, Lee MW, Choi D, Lee WJ, et al. Ten-year outcomes of percutaneous radiofrequency ablation as first-line therapy of early hepatocellular carcinoma: analysis of prognostic factors. J Hepatol 2013;58:89-97.

4. Lee DH, Lee JM, Lee JY, Kim SH, Yoon JH, Kim YJ, et al. Radiofrequency ablation of hepatocellular carcinoma as first-line treatment: long-term results and prognostic factors in 162 patients with cirrhosis. Radiology 2014;270:900-909.

5. Jang MK, Lee HC, Kim IS, Lim YS, Chung YH, Lee YS, et al. Role of additional angiography and chemoembolization in patients with hepatocellular carcinoma who achieved complete necrosis following transarterial chemoembolization. J Gastroenterol Hepatol 2004;19:1074-1080.

6. Kim JH, Sinn DH, Shin SW, Cho SK, Kang WS, Gwak GY, et al. A role of scheduled second TACE in early stage hepatocellular carcinoma showing complete response after first TACE. Clin Mol Hepatol 2017;23:42-51.

7. Miyayama S, Matsui O, Yamashiro M, Ryu Y, Kaito K, Ozaki K, et al. Ultraselective transcatheter arterial chemoembolization with a $2-f$ tip microcatheter for small hepatocellular carcinomas: relationship between local tumor recurrence and visualization of the portal vein with iodized oil. J Vasc Interv Radiol 2007;18:365-376. 
Jin Wook Chung

Chemoembolization for HCC; scheduled vs on-demand

8. Kang HJ, Kim YI, Kim HC, Jae HJ, Hur S, Chung JW. Does establishing a safety margin reduce local recurrence in subsegmental transarterial chemoembolization for small nodular hepatocellular carcinomas? Korean J Radiol 2015;16:1068-1078. 